\title{
Silica through the eyes of colloidal models-when glass is a gel
}

\author{
Ivan Saika-Voivod ${ }^{1}$, Heather Marie King ${ }^{1}$, Piero Tartaglia ${ }^{2}$, \\ Francesco Sciortino ${ }^{2}$ and Emanuela Zaccarelli ${ }^{2}$ \\ ${ }^{1}$ Department of Physics and Physical Oceanography, Memorial University of Newfoundland, \\ St John's, NL, A1B 3X7, Canada \\ ${ }^{2}$ Dipartimento di Fisica and CNR-ISC, Università di Roma La Sapienza, \\ Piazzale Aldo Moro 2, 00185 Rome, Italy
}

Received 10 February 2011, in final form 6 May 2011

Published 10 June 2011

Online at stacks.iop.org/JPhysCM/23/285101

\begin{abstract}
We perform molecular dynamics simulations of 'floating bond' (FB) models of network-forming liquids and compare the structure and dynamics against the BKS model of silica (van Beest et al 1990 Phys. Rev. Lett. 64 1955), with the aim of gaining a better understanding of glassy silica in terms of the variety of non-ergodic states seen in colloids. At low densities, all the models form tetrahedral networks. At higher densities, tailoring the FB model to allow a higher number of bonds does not capture the structure seen in BKS. Upon rescaling the time and length in order to compare mean squared displacements between models, we find that there are significant differences in the temperature dependence of the diffusion coefficient at high density. Additionally, the FB models show a greater range in variability in the behavior of the non-ergodicity parameter and caging length, quantities used to distinguish colloidal gels and glasses. Hence, we find that the glassy behavior of BKS silica can be interpreted as a 'gel' at low densities, with only a marginal gel-to-glass crossover at higher densities.
\end{abstract}

(Some figures in this article are in colour only in the electronic version)

\section{Introduction}

In recent years, researchers have introduced simplified models to better understand the essential elements behind the unconventional thermodynamic and dynamic properties of silica, water and tetrahedral network-forming liquids in general, as well as the differences between them, such as why silica is such a good glass-former, while water readily crystallizes. Compared to atomistic models such as the BKS model of silica [1] and the SPC/E model for water [2], these simpler models condense the long-range electrostatic interactions into short-range ones but, following the pioneering work of Kolafa and Nezbeda on water [3], explicitly enforce a tetrahedral valence by decorating hard spheres with four sticky spots for water or one-component tetrahedral particles [3-5], or by employing a 1:2 mixture of spheres with four and two sticky spots representing $\mathrm{Si}$ and $\mathrm{O}$ atoms in silica $[6,7]$.

Interestingly, these types of simpler models have recently become relevant for colloidal systems, especially in the framework of newly synthesized patchy particles [8-11] or for functionalized particles, either via DNA-binding [12-14] or through specific attraction $[15,16]$. Networks can also be studied by simulating binary mixtures of particles interacting with isotropic interactions, where one species provides the network nodes and the other one acts as a collection of 'floating bonds' (FBs) [17-20]. Tetrahedrality is attained by stoichiometry and a judicious choice of the potential parameters, and could potentially be achieved experimentally with mixtures of oppositely charged particles [21].

Recently, it was noticed that generic four-valence models, including primitive models of water [3] and silica [6], DNA dendrimers [12], the limited valency model [22, 23], as well as the FB model [18, 19], share a similar phase diagram [24] both in terms of the location of the gas-liquid spinodal and in the behavior of the iso-diffusivity lines, once temperature and density are correctly scaled respectively to the critical temperature and to the number density of network nodes. This phase diagram was also compared to those of well-studied models of water (SPC/E and ST2) as well as to BKS silica, again showing striking similarities [24]. Building on this 
correspondence, we provide in this article a direct comparison between the more realistic and well-studied model for silicathe BKS model-and two simpler FB models, mimicking tetrahedral-forming colloidal particles.

The BKS model consists of short-range pairwise additive forces along with long-range Coulomb interactions, and reproduces to varying degrees of accuracy the physical properties of silica we are seeking to understand with (shortrange) FB models. While the coefficients used in the BKS potential can be refined to improve its comparison with experiments $[25,26]$ (indeed BKS is an improvement over the TTAM potential [27]) and further improvements can be achieved through adding three-body forces and variable charges [28], the BKS model has become a standard for computational studies of the static and dynamic properties of silica [29, 30], and serves as a point of comparison for further improvements [31]. Modifications to BKS-like potentials have shed insight into the physics of the network-forming liquids $\mathrm{MX}_{2}$, such as was done in [32], in which the authors connected anion polarizability, $\mathrm{M}-\mathrm{X}-\mathrm{M}$ bond angle, local and intermediate-range order and glass-forming fragility.

Modifications to the Stillinger-Weber model of silicon [33], a monatomic tetrahedral liquid, have also shed light on the importance of bond angle and local coordination constraints on phase behavior, glassy dynamics, gel formation and liquid-liquid phase separation [34-36]. However, we find it more expedient to initially map FB models onto BKS (a mixture), rather than to a system with explicit three-body interactions.

By directly comparing structural and dynamical properties of $\mathrm{BKS}$ and $\mathrm{FB}$ models, we want to investigate whether the similarity of phase diagrams can be pushed beyond a qualitative agreement. In particular, we focus on the study of the different systems close to the arrest line at several densities, in order to establish a connection between silica glass, which is the prototype of a 'strong' glass-former, and the variety of non-ergodic states that are found in colloidal systems, in particular gels, as well as attractive and repulsive glasses [37-39]. Indeed, upon increasing the density, silica is known to become a more 'fragile' glass-former, and it would be interesting to connect this transition to that between a (strong) gel and a (fragile) glass [24, 40].

We note that in this paper we are referring to equilibrium gels [37], where the bond strength is of the order of the thermal energy. Although in silica the bonds possess a covalent character, we are studying them at a temperature scale for which the bonds can form reversibly and have a finite lifetime.

The first model (FBH, floating bond hard), described in detail in [18], is based on (non-additive) hard-core repulsions and square well attractions of depth $u_{0}$, with attraction only between particles of different types. In FBH, the effective directional attraction gives rise to tetrahedral structure in a limited window of densities, the so-called optimal networkforming region, where at low temperatures the system develops a fully bonded tetrahedral network. The second model (FBS, floating bond soft), introduced here, replaces the non-additive hard sphere interaction between floating bonds of model FBH with a finite shoulder and a smaller hard core, to allow for network interpenetration and increased coordination, as occurs in silica at higher densities.

For simplicity, we focus on two fluid isochores at either end of the density $\rho$ range of BKS. The first isochore, at $\rho_{\text {low }}=2.3 \mathrm{~g} \mathrm{~cm}^{-3}$ (near the ambient density of real silica), is located in the optimal network region, close to the liquidgas spinodal [41], where at low temperature $T$ the tetrahedral network forms and the dynamics is becoming strong. The second isochore is located at $\rho_{\text {high }}=3.9 \mathrm{~g} \mathrm{~cm}^{-3}$, where the liquid is no longer tetrahedral, exhibits fragile glassy dynamics, is on the high density side of both the diffusivity maximum and any possible liquid-liquid critical point occurring in the model [42] and is approaching the density where spontaneous crystallization to stishovite prevents us from deeply probing the supercooled regime [43]. In this way we explore the largest variation, in terms of density, of the slowing down of supercooled liquid silica and we try to connect our findings with the behavior of colloidal systems.

In the remainder of this paper, we present our analysis of the models, discuss the results and finish with conclusions and ideas for future work.

\section{Models and simulations}

The FBH model was initially studied in $[18,19]$. The FBS model, introduced here, also consists of $N_{\mathrm{p}}=1000$ 'node' particles (modeling Si atoms, labeled 1) that interact with each other via a hard sphere interaction of diameter $\sigma$, and 2000 'floating bonds' ( $\mathrm{O}$ atoms, labeled 2). For both FBH and FBS, particles of types 1 and 2 interact through a short-range square well

$$
V_{12}(r)= \begin{cases}\infty & r<\sigma_{12} \\ -u_{0} & \sigma_{12}<r<\sigma_{12}+\delta \\ 0 & r>\sigma_{12}+\delta,\end{cases}
$$

where $\sigma_{12}=0.55 \sigma$, and $\delta /(1+\delta)=0.03 \sigma_{12}$. For FBH, type 2 particles interact with a hard sphere repulsion of diameter $\sigma_{22}=0.8 \sigma$. For FBS, the potential between particles of type 2 is softened to a finite shoulder via

$$
V_{22}^{\mathrm{S}}(r)= \begin{cases}\infty & r<\sigma_{\text {small }} \\ u_{0} & \sigma_{\text {small }}<r<\sigma_{22} \\ 0 & r>\sigma_{22},\end{cases}
$$

where $\sigma_{\text {small }}=0.1 \sigma$. Both types of particle have mass $m$. The FBH and FBS models are studied by extensive event-driven molecular dynamics simulations.

A detailed comparison between BKS and the FB models requires a mapping of the length and time scales. In the following we use the network node-node distance $\sigma_{\text {eff }}$ (the equivalent of the $\mathrm{Si}-\mathrm{Si}$ distance in BKS), taken to be the location of the first peak in the $\mathrm{Si}-\mathrm{Si}$ partial radial distribution function, as a unit of length. For BKS, $\sigma_{\text {eff }}=0.31 \mathrm{~nm}$ and for the FB models $\sigma_{\text {eff }}=1.05 \sigma$. With this unit, the scaled number densities $\rho^{*}=N_{\mathrm{p}} \sigma_{\text {eff }}^{3} / V$ (again, $N_{\mathrm{p}}$ is the number of node ( $\mathrm{Si}$ ) particles and $V$ is the volume) for the BKS isochores are $\rho_{\text {low }}^{*}=0.69$ and $\rho_{\text {high }}^{*}=1.16$. We also scale time by $t_{\text {eff }}$, the approximate time at which the ballistic motion of the node atoms crosses to diffusive motion. For BKS 




Figure 1. Si-Si partial structure factors $S(q)$ for BKS silica at (a) low and (b) high density, compared with FB models; parentheses indicate $\left(\rho^{*}, T^{*}\right)$ pairs. $\sigma_{\text {eff }}$ is the position of the first peak of the $\mathrm{Si}-\mathrm{Si}$ (node-node) radial distribution function: for BKS $\sigma_{\text {eff }}=0.31 \mathrm{~nm}$; for FBH and FBS $\sigma_{\text {eff }}=1.05 \sigma(\sigma$ is the node particle diameter).

silica, this corresponds to $t_{\mathrm{eff}}=75 \mathrm{fs}$. For the FB models, $t_{\text {eff }}=\sigma \sqrt{m / u_{0}}$, i.e. simply the usual definition of unit time. The temperature for the FB models is reported in reduced units, $T^{*}=k_{\mathrm{B}} T / u_{0}$.

In order to compare the structure and dynamics, we report the following quantities: the static structure factor $S(q)=$ $\left\langle\rho_{q}(0) \rho_{-q}(0)\right\rangle$, where $\rho_{\vec{q}}(t)=N_{\mathrm{p}}^{-1 / 2} \sum_{i=1}^{N_{\mathrm{p}}} \exp \left[-\mathrm{i} \vec{q} \cdot \vec{r}_{i}(t)\right]$; the mean square displacement (MSD) of node particles along with its associated caging length $l_{0}$, defined as the square root of the height of the MSD plateau prior to the onset of diffusive behavior; the non-ergodicity parameter $f_{q}$ and stretching exponent $\beta_{q}$, both of which are obtained from a fit of the secondary relaxation portion of the intermediate scattering function $F(q, t)=\left\langle\rho_{q}(t) \rho_{-q}(0)\right\rangle / S(q)$ to the fitting function $f_{q} \exp \left[-\left(t / \tau_{q}\right)^{\beta}\right]$. All quantities are based on node particles alone, $\mathrm{Si}$ atoms in the case of BKS silica and the larger particles in the FBH and FBS systems.

\section{Results}

In figure 1(a) we compare $S(q)$ for low $T$ and low density for $\mathrm{BKS}$ and FBH. At low density, $S(q)$ for FBS is not discernible from $\mathrm{FBH}$, and is therefore not shown. The pre-peak in $S(q)$ displayed by BKS silica at $\rho_{\text {low }}^{*}$ is a signature of the developed tetrahedral network. It is also found in the FB models at $\rho^{*}=0.66$, with a slightly lower intensity. Hence in terms of static structure, there seems to be quite a good correspondence between the models (also in terms of $\rho^{*}$ ).

At higher density the situation is different, as illustrated in figure 1(b). Now the BKS structure is very different from that of either FBH or FBS near comparable densities. Indeed, the splitting of the first $S(q)$ peak in BKS persists up to high density despite six-fold coordination, suggesting the presence of a locally ordered octahedral network. On the other hand, in the FB models, geometrical preference is lost with increasing density, even in the case of FBS, where the model would allow for the formation of six bonds per particle.

To compare the FB and BKS dynamics, we begin by finding state points in FBS and FBH that match the mean square displacement of BKS at the lowest $T$ for both isochores, both in terms of diffusion coefficient and plateau height. In figure 2(a), we see that for $\rho_{\text {low }}$ at $T=3000 \mathrm{~K}$, the MSD is matched by both FBH and FBS at $\left(\rho^{*}, T^{*}\right)=(0.88,0.10)$ (a density considerably larger than $\rho_{\text {low }}^{*}$ ), while for $\rho_{\text {high }}$ at $T=2400 \mathrm{~K}$, the MSD is matched by $\mathrm{FBH}$ at $\left(\rho^{*}, T^{*}\right)=$ $(1.05,0.15)$, and by FBS at $(1.15,0.15)$. This matching procedure operated at the lowest BKS studied temperature provides us with a conversion value to compare the full $T$ dependence of the dynamics in the different models. It is found that such a procedure implicitly assumes a conversion factor for $T$ which is density dependent, providing the scaling factors $T_{\mathrm{S}}=3000 / 0.1$ at $\rho_{\text {low }}^{*}$ and $T_{\mathrm{S}}=2400 / 0.15$ at $\rho_{\text {high }}^{*}$. In this way, we report in figure 2(b) the $T$ dependence of the self-diffusion coefficient $D$, evaluated from the long time limit of the MSD, normalized by $\sigma_{\text {eff }}^{2} / t_{\text {eff }}$. The FB models at $\rho^{*}=0.88$, at low $T$, match the $\rho_{\text {low }}^{*}$ BKS isochore quite well, being nearly parallel for more than two orders of magnitude in $D$. For the $\rho_{\text {high }}^{*}$ BKS isochore, the agreement with the FB models is worse. While in BKS the $T$-dependence of $D$ is non-Arrhenius, $D$ for the FB models shows a clear Arrhenius dependence at low $T$. The two FB models show fairly good agreement with each other.

Figure 3 shows the caging length as a function of density along the isotherms $T=3000 \mathrm{~K}$ for BKS and $T^{*}=0.10$ for the FB models. It is evident that $\rho^{*} \simeq 0.88$ marks the threshold above which the two FB models become different. Above this value, the soft-core interactions enter into play as the packing increases, although, as we have seen in figure 1, the structures remain quite similar. It is quite clear that for similar changes in density, BKS shows much less change in $l_{0}$ than the FB models. The similarity between FBH and FBS is highlighted in the inset of figure 3, where we show the pressure dependence of $l_{0}$. While the two FB curves collapse, BKS shows a strong departure. Here, in order to compare the models, we have reduced the pressures by a factor $m_{\mathrm{Si}} /\left(t_{\text {eff }}^{2} \sigma_{\text {eff }}\right)$, which is $2.68 \times 10^{10} \mathrm{~Pa}$ for silica. We note that the BKS model exhibits negative pressures for densities near $\rho_{\text {low }}$ at $3000 \mathrm{~K}$ - a drawback that can be addressed by reparameterizing the potential $[25,26]$.

We now try to gain a deeper understanding of the nature of the dynamical arrest occurring at different densities, viewing silica through the eyes of colloidal systems. Indeed, as alluded to in the beginning, the FB models could perhaps be realized with colloidal particles. The behavior of $l_{0}$, extracted from the MSD, already indicates an increasing length scale associated with arrest as the density decreases. To better characterize the underlying arrest mechanisms in terms of the different length scales, it is instructive to monitor the so-called non-ergodicity parameter $f_{q}$, i.e. the plateau height of the collective density correlation function. This typically oscillates in phase with $S(q)$ and is non-zero in a finite range of $q$, providing direct evidence of the characteristic length scales over which the system becomes non-ergodic. While for a standard glass $f_{q}$ 

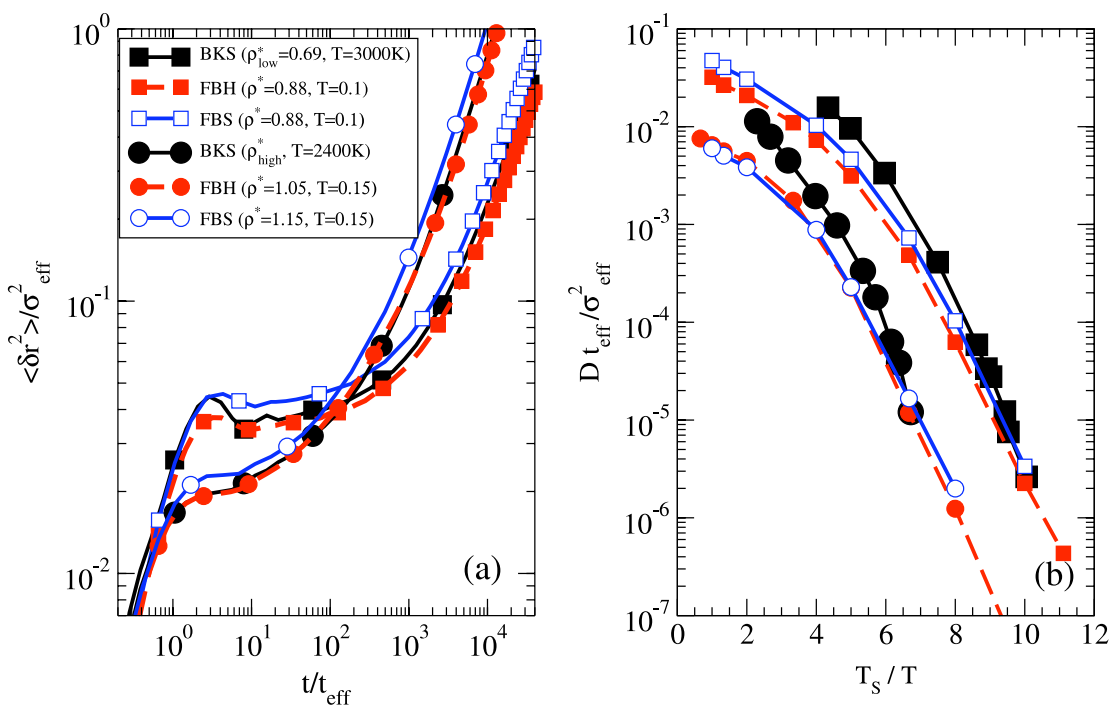

Figure 2. Dynamics of BKS and FB models, characterized by (a) the MSD (parentheses indicate $\left(\rho^{*}, T^{*}\right)$ pairs), (b) the $T$ dependence of the diffusion coefficient (density given by symbols as in (a); $T_{\mathrm{S}}=30000 \mathrm{~K}$ for $\rho_{\text {low }}$ and $16000 \mathrm{~K}$ for $\rho_{\text {high }}$ for BKS, in order to match state points in (a)).

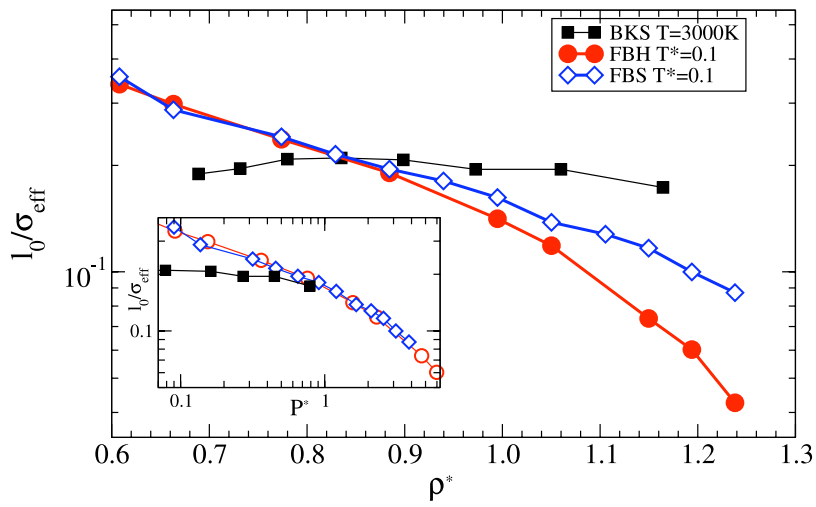

Figure 3. Caging length $l_{0}$ along isotherms for BKS, FBH and FBS. The inset shows $l_{0}$ as a function of reduced pressure $P^{*}=P t_{\mathrm{eff}}^{2} \sigma_{\mathrm{eff}} / m$.

is significantly different from zero at all physically relevant $q$ values, implying that all length scales are arrested, gels exhibit a very different behavior $[22,44]$. Indeed, $f_{q}$ is finite only at small $q$, providing evidence that only large length scales, typically of the order of the mesh of the gel network, are arrested, while on smaller scales (including nearest-neighbor ones) the system remains ergodic. Previous studies have also shown that, with increasing density, a gel-to-glass crossover is observed, signaled by a growth of $f_{q}$ at all $q$ [40].

We now ask whether we can classify arrested states of silica as gel or glass by comparing the behavior of $f_{q}$ to that of the FB models. We also monitor the behavior of the stretching exponents $\beta_{q}$. Colloidal gels can be viewed [24] as the counterpart of strong glasses ( $\beta_{q}$ nearer to 1) [45], while colloidal glasses are similar to fragile glasses $\left(\beta_{q}\right.$ smaller than 1).

Figure 4 shows $f_{q}$ for state points that match the MSD in $\mathrm{BKS}, \mathrm{FBS}$ and FBH (the same as in figure 2(a)). We see that

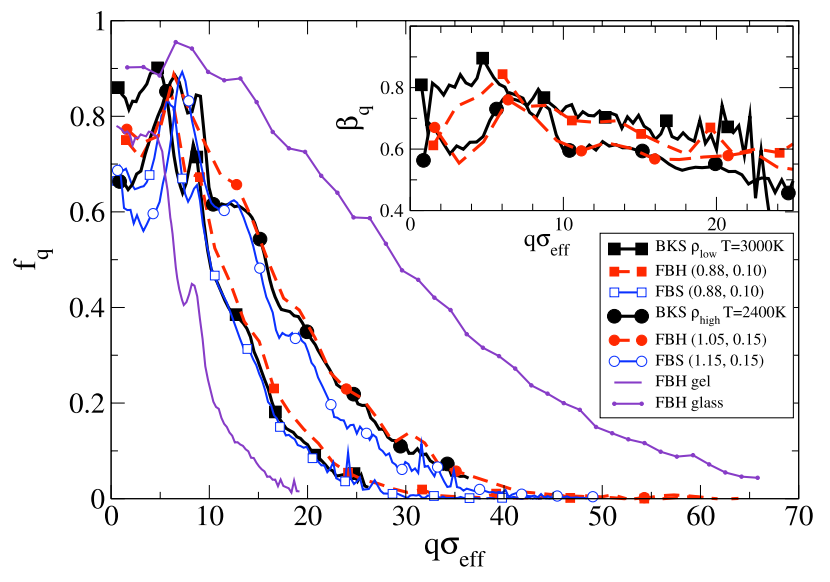

Figure 4. The non-ergodicity parameter for BKS and FB models. The signature gel and glass curves $(\mathrm{FBH})$ are taken from $\left(\rho^{*}, T^{*}\right)=(0.66,0.09)$ and $(1.15,0.09)$, respectively. Inset: stretching exponent.

for both $\rho_{\text {low }}^{*}$ and $\rho_{\text {high }}^{*}$, a match in the MSD corresponds also to a match in terms of $f_{q}$ and $\beta_{q}$. Also shown in figure 4 are $f_{q}$ curves for $\mathrm{FBH}$ at $\rho^{*}=0.66$ and 1.15 , in order to underline the dramatic variation in the range of $f_{q}$ over a similar range in density as chosen for BKS. These two curves illustrate the signatures of gels and glasses in terms of $f_{q}$. The softening of the non-additive repulsion between floating bonds in FBS reduces the extent of $f_{q}$ to that seen in BKS at a comparably high density. As the density is lowered, BKS does not achieve the same degree of narrowing of $f_{q}$ as the FB models. Thus we can conclude from figure 4 that if we can associate a narrow $f_{q}$ with gels and an extended one with repulsive glasses, the 'gel-to-glass' crossover is much more muted in BKS. However, using FBS does shift the colloidal model toward the behavior of BKS. 


\section{Discussion}

In the present paper, we compare BKS silica and two floating bond models, one that maintains tetravalent bonding to high densities $(\mathrm{FBH})$ and one that allows more bonds to form as the density increases (FBS). The modifications present in FBS only seem to matter beyond $\rho^{*}=0.88$; at lower $\rho^{*}$ the two models are practically the same.

At comparably low reduced number densities, $\rho_{\text {low }}^{*}=0.69$ for BKS and $\rho^{*}=0.66$ for both FBS and FBH, tetrahedral order is present in all models. However, in terms of dynamics, as shown by the caging length and the shape of the MSD, a better match to $\rho_{\text {low }}^{*}$ BKS is attained at $\rho^{*}=0.88$ in the FB models, by which point the structure factors for the FB models are considerably different from BKS. Nonetheless, the $T$ dependence of the dynamics of FBH and FBS at $\rho^{*}=0.88$, as presented through $D$, is quite similar to that of BKS at $\rho_{\text {low }}^{*}$ : see figure 2(b), upper curves, where the slopes of the curves are not that different. At high density, BKS silica is quite non-Arrhenius while the FB models retain Arrhenius dynamics, indicating that the energetic barrier to diffusion is still increasing in BKS, while for the FB models it is well defined by the square well bond energy.

We also see that in the regime where the FB models form a tetrahedral network structure, the caging length is too large in comparison to BKS. Our understanding of the relationship between the parameters of the model and the caging length (or the degree of splitting of the first peak in $S(q)$ ) is lacking, and thus would naturally be a target of future study.

As a function of density, the caging length of BKS is fairly invariant compared to the range seen for the FB models. It is interesting to note that while BKS does show a diffusivity anomaly within the studied density range, $l_{0}$ along a curve of constant $D$ is monotonic; it appears that the caging length is not directly coupled to $D$ across the density. While FBS does exhibit less localization (larger $l_{0}$ ) at higher $\rho^{*}$ than FBH, its range in $l_{0}$ is still quite broad compared to BKS.

One motivation for this work was the idea of comparing glassy BKS to the non-ergodic states seen in colloidal models, primarily the low density gel and the high density repulsive glass. The dynamic signatures of gels include a large $l_{0}$, a small range in $q$ for which $f_{q}$ is appreciably non-zero and a value of the stretching exponent $\beta_{q}$ close to unity. Conversely, repulsive glasses have a short $l_{0}$, a large range of $f_{q}$ and $\beta_{q}$ closer to $1 / 2$. We find that while BKS does have a gel-to-repulsive glass trend, at least in terms of the range of $f_{q}$ and values of $\beta_{q}$, the effect is relatively small compared to FBH, or even to FBS.

Thus, while glassy silica can be interpreted as a 'gel' at low density, it does not show a clear crossover to a glass on increasing $\rho$, i.e., the behaviors of $l_{0}$ and $f_{q}$ are much less dependent on density than in colloidal systems. As a first step toward a better understanding of this difference, FBS does exhibit a reduced density dependence of these quantities.

\section{Conclusions}

In this paper, we have exploited the similarity of the phase diagrams for generic tetrahedral models [24] in order to connect silica to simpler models relevant to colloidal systems. Working with reduced quantities, we find that the structure of the FB models maps fairly well to that of BKS at low density. However, there seems to be a mismatch in the density at which the dynamics are most similar.

The trend in the dynamics for BKS in going from low to high density is suggestive of the crossover from gel-torepulsive glass seen in colloidal models, i.e. $f_{q}$ broadens in its extent, $\beta_{q}$ decreases although $l_{0}$ remains rather constant. However, the trend is not striking considering the behavior FBH and FBS exhibit over a similar range in $\rho^{*}$. While replacing the hard sphere repulsion with a shoulder in the FB model does not lead to anomalous behavior in density or diffusivity, it does change the dynamics (and the pressure) beyond $\rho^{*} \approx 0.88$ significantly, while only subtly changing the structure. The reduced pinning of tetrahedral centers provided by the shoulder brings FBS closer to the dynamical behavior of BKS, even though the splitting of the first peak in $S(q)$ is lost. The non-Arrhenius behavior of $D$ for BKS suggests a still-changing energy landscape at low $T$, particularly at $\rho_{\text {high }}$, which could be better captured by even softer FB models.

It would be interesting to know to what extent the lack of strong gel-to-glass signatures and the appearance of non-Arrhenius dynamics can be captured by models with short-range interactions, or whether longer-range forces are necessary.

\section{Acknowledgments}

We thank S V Buldyrev for useful discussions and ACEnet, SHARCNET, NSERC, ERC-226207-PATCHYCOLLOIDS and ITN-234810-COMPLOIDS for computing resources and funding.

\section{References}

[1] van Beest B W H, Kramer G J and van Santen R A 1990 Phys. Rev. Lett. 641955

[2] Berendsen H J C, Grigera J R and Straatsma T P 1987 J. Phys. Chem. 91 6269-71

[3] Kolafa J and Nezbeda I 1987 Mol. Phys. 61161

[4] Bianchi E, Largo J, Tartaglia P, Zaccarelli E and Sciortino F 2006 Phys. Rev. Lett. 97168301

[5] Bianchi E, Tartaglia P, Zaccarelli E and Sciortino F 2008 J. Chem. Phys. 128144502

[6] Ford M H, Auerbach S M and Monson P A 2004 J. Chem. Phys. 1218415

[7] De Michele C, Tartaglia P and Sciortino F 2006 J. Chem. Phys. 1254710

[8] Manoharan V N, Elsesser M T and Pine D J 2003 Science $301483-7$

[9] Zhang G, Wang D and Möhwald H 2005 Angew. Chem. Int. Edn 44 1-5

[10] Glotzer S C and Solomon M J 2007 Nat. Mater. 8 557-62

[11] Kraft D J, Groenewold J and Kegel W K 2009 Soft Matter 53823

[12] Starr F W and Sciortino F 2006 J. Phys.: Condens. Matter 18 L347-53

[13] Nykypanchuk D, Maye M M, van der Lelie D and Gang O 2008 Nature 451549

[14] Schmatko T, Bozorgui B, Geerts N, Frenkel D, Eiser E and Poon W C K 2007 Soft Matter 3703 
[15] Hiddessen A L, Rodgers S D, Weitz D A and Hammer D A 2000 Langmuir 16 9744-53

[16] Corezzi S, De Michele C, Zaccarelli E, Fioretto D and Sciortino F 2008 Soft Matter 41173

[17] Ferrante A and Tosi M P 1989 J. Phys.: Condens. Matter 1 1679-94

[18] Zaccarelli E, Tartaglia P and Sciortino F 2007 J. Chem. Phys. 127174501

[19] Mayer C, Sciortino F, Tartaglia P and Zaccarelli E 2010 J. Phys.: Condens. Matter 22104110

[20] Coslovich D and Pastore G 2009 J. Phys.: Condens. Matter 21285107

[21] Leunissen M E, Christova C G, Hynninen A-P, Royall C P, Campbell A I, Imhof A, Dijkstra M, van Roij R and van Blaaderen A 2005 Nature 437235

[22] Zaccarelli E, Buldyrev S V, La Nave E, Moreno A J, Saika-Voivod I, Sciortino F and Tartaglia P 2005 Phys. Rev. Lett. 94218301

[23] Moreno A J, Buldyrev S V, La Nave E, Saika-Voivod I, Sciortino F, Tartaglia P and Zaccarelli E 2005 Phys. Rev. Lett. 95157802

[24] Sciortino F 2008 Eur. Phys. J. B 64 505-9

[25] Carré A, Horbach J, Ispas S and Kob W 2008 Europhys. Lett. 8217001

[26] Horbach J 2008 J. Phys.: Condens. Matter 20244118

[27] Tsuneyuki S, Tsukada M, Aoki H and Matsui Y 1988 Phys. Rev. Lett. 61869

[28] Huang L, Duffrene L and Kieffer J 2004 J. Non-Cryst. Solids 3491
[29] Horbach J and Kob W 1999 Phys. Rev. B 603169

[30] Shell M S, Debenedetti P G and Panagiotopoulos A Z 2002 Phys. Rev. E 66011202

[31] Kerrache A, Teboul V and Monteil A 2006 Chem. Phys. 32169

[32] Wilson M and Salmon P S 2009 Phys. Rev. Lett. 103157801

[33] Stillinger F H and Weber T A 1985 Phys. Rev. B 315262

[34] Molinero V, Sastry S and Angell C A 2006 Phys. Rev. Lett. 97075701

[35] Saw S, Ellegaard N L, Kob W and Sastry S 2009 Phys. Rev. Lett. 103248305

[36] Saw S, Ellegaard N L, Kob W and Sastry S 2011 J. Chem. Phys. 134164506

[37] Zaccarelli E 2007 J. Phys.: Condens. Matter 19323101

[38] Sciortino F and Tartaglia P 2005 Adv. Phys. 54 471-524

[39] Chaudhuri P, Berthier L, Hurtado P I and Kob W 2010 Phys. Rev. E 81 040502R

[40] Zaccarelli E, Saika-Voivod I, Moreno A J, Buldyrev S V, Tartaglia P and Sciortino F 2006 J. Chem. Phys. 124124908

[41] Guisanni Y and Guillot B 1996 J. Chem. Phys. 1047633

[42] Saika-Voivod I, Sciortino F and Poole P H 2001 Phys. Rev. E 63011202

[43] Saika-Voivod I, Sciortino F and Poole P H 2004 Phys. Rev. E 69041503

[44] Saika-Voivod I, Zaccarelli E, Sciortino F, Buldyrev S V and Tartaglia P 2004 Phys. Rev. E 70041401

[45] Böhmer R, Ngai K L, Angell C A and Plazek D J 1993 J. Chem. Phys. 994201 